\title{
Estructuraciones para analizar realidades complejas
}

\author{
Prof. Ridberth M. Ramírez Miranda
}

\section{RESUMEN}

El conocimiento de arquitecturas conceptuales hace que podamos observar críticamente lo que ocurre en la realidad, para adoptar una posición científica y usar una metodología adecuada en la investigación, de manera que algunas de ellas puedan servir para inducir límites a la arbitrariedad de la investigación, para visualizar la estructura de los problemas o para promover ideas, tanto correctoras como creativas, con el objetivo de caracterizar un paradigma de lo complejo, relativamente poco conocido, en el campo de las ideas posibles, así como una evaluación general de complejidad y caos.

El fenómeno de la globalización toca a las universidades, persuadiéndolas de hacer un mayor esfuerzo en entender y desarrollar conocimiento útil y pertinente para los procesos productivos. Siendo así, es indefectible una alianza social entre las universidades, las empresas y el Estado para incorporar conocimiento, innovación y diseñar e implementar estrategias coherentes para competir en los mercados nacionales y mundiales.

La iniciativa debe ser fundamentalmente conformar equipos científicos con representantes de las universidades, las empresas y el Estado, para proponer estrategias que contribuyan al desarrollo de la ciencia y la tecnología en el país.

Palabras clave: Estructucciones

\section{ABSTRACT}

Knowledge of conceptual architectures makes us look critically at what happens in 


\section{Ridberth M. Ramírez Miranda}

reality, to adopt a scientific position and use it in a adequate methodology in searching, so, any of them can lead to limits on the arbitrariness of the investigation, to visualize the structure of problems or to promote ideas, both corrective and creative, the goal is to describe a paradigm of the complex, relatively little known, in the field of possible ideas, as well as an overall assessment of complexity and chaos.

Globalization comes to the universities, persuading them in making a greater effort to understand and develop useful and relevant knowledge to the production processes. Therefore, it is an unfailing social partnership between universities, businesses and the state to incorporate knowledge, innovation and design and implement coherent strategies to compete in domestic and global markets.

The initiative must be primarily scientific teams up with representatives from universities, corporations and the State, to propose strategies that contribute to the development of science and technology in the country.

\section{INTRODUCCION}

Uno de los temas difíciles de abordar en toda investigación son los comportamientos sociales complejos, en la relación Universidad, Empresa y Estado; muchos de los estudiosos los han abordado asumiendo una "complejidad organizada" que propone una fundamentación razonablemente sólida de los métodos para abordar los fenómenos complejos, para lo que se revisa en detalle las estructuraciones mas representativos ligados a la complejidad.

Se plantea críticamente la posibilidad de aplicar arquitecturas conceptuales a la realidad empírica de la que se ocupa la investigación, de manera que alguno de ellos puede servir para inducir límites a la arbitrariedad de la investigación, para visualizar la estructura de los problemas o para promover ideas, tanto correctoras como creativas.

El objetivo es caracterizar un paradigma de lo complejo que ocupa una región particular, relativamente poco conocida, en el campo de las ideas posibles, una evaluación general de complejidad y caos, digamos que:

- Contiene estructuras resumidas y precisas sobre temas de las ciencias sociales, teorías científicas y recursos; sería una puerta adecuada para entrar de manera clara y 


\section{Estructuraciones para analizar realidades complejas}

documentada al amplio paradigma de lo complejo.

- Es una lógica para quien busque profundizar la discusión entre ciencia, humanidades y corrientes interpretativas, o quiera tener un panorama de las investigaciones complejas en ciencias sociales.

- El criterio es por la incorporación de nuevas ideas a la investigación en las Ciencias Sociales, desde una postura de que hay una realidad afuera, que esa realidad puede y debe ser cambiada.

En suma, es un planteamiento sobre la investigación en complejidad y Ciencias Sociales, en la triada Universidad, Empresa y Estado. Habrá que esperar un tiempo para saber de su difusión y validez entre los investigadores de la universidad y llegue a los estudiantes y jóvenes profesionales de las ciencias sociales, que podrán ser los que mas usen las nuevas herramientas disponibles.

Consecuentemente, a partir de esta lógica, plantearemos tres criterios fundamentales para el análisis de los comportamientos complejos en las Ciencias Sociales:

\section{EL CRITERIO DEL CONOCIMIENTO}

En este punto el detalle es como se estructura la aplicabilidad de la teoría y los instrumentos generados por la ciencia a una realidad concreta, de manera que signifique una secuencia, la misma que se legitima con la estructura: teoría, instrumentos y realidad.

\subsection{Teoría}

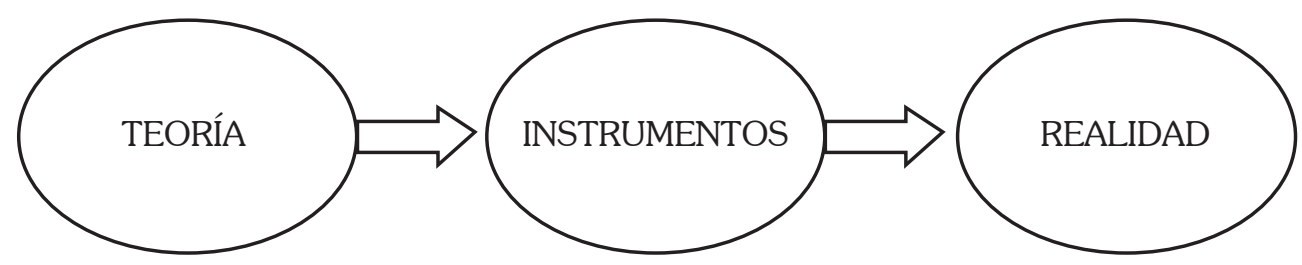




\section{Ridberth M. Ramírez Miranda}

Una teoría es un sistema lógico compuesto de observaciones, axiomas y postulados, que tienen como objetivo declarar bajo qué condiciones se desarrollarán ciertos supuestos; a partir de estas, se pueden especular, deducir y/o postular mediante ciertas reglas o razonamientos, otros posibles hechos en el campo de la investigación.

Es un sistema hipotético-deductivo que constituye una explicación o descripción científica a un conjunto relacionado de observaciones o experimentos, de una realidad concreta. Es un modelo para el entendimiento de un conjunto de hechos empíricos formalizado por una infraestructura matemática capaz de producir predicciones experimentales para una realidad dada.

Formalmente una teoría es un sistema conceptual, comprensivo y explicativo; racional, objetivo y empírico, sobre hechos o algún aspecto de la realidad

Abarca, en general, varias leyes científicas verificadas y en ocasiones deducibles de la propia teoría. Estas leyes pasan a formar parte de los supuestos e hipótesis básicas de la teoría que englobará los conocimientos aceptados por la comunidad científica del campo de investigación y está aceptada por la mayoría de investigadores.

\subsection{Instrumentos}

Los instrumentos científicos han suscitado tradicionalmente el interés de todos los que han pretendido entender las características de la actividad científica. Un objeto se transforma en instrumento científico por el uso, es decir, adquiere esta condición por el hecho de ser utilizado en una investigación científica.

Algunos diferencian entre instrumentos pasivos, destinados a la observación y a la medida, e instrumentos activos, cuyo propósito es la creación de nuevos fenómenos en el laboratorio. Todo esto permite diferenciar entre instrumentos destinados a la investigación, a la docencia o a los usos industriales.

En realidad, un mismo instrumento puede pasar de un contexto a otro y servir 


\section{Estructuraciones para analizar realidades complejas}

de esta manera de "mediador" entre ciencia e industria o entre diferentes disciplinas científicas, siendo necesario que las comunidades científicas los acepten como un medio seguro para realizar investigaciones.

Como vemos, los instrumentos científicos se pueden analizar desde múltiples perspectivas que permiten convertirlos en nexos entre diferentes disciplinas. De esta manera, pueden servir para ofrecer una imagen mucho más humana de la actividad científica y actuar como puentes entre las Ciencias Sociales y las Ciencias Naturales.

\subsection{La ciencia y los instrumentos científicos}

Los progresos científicos dependen, como el efecto de la causa, del desarrollo de los instrumentos científicos.

Se puede decir, en general, que el progreso de las ciencias, es el producto de la utilización de los principales "instrumentos" del progreso científico y son de orden intelectual (y también político), metodológico; los "instrumentos intelectuales" no surgieron de la nada, no son innatos en el hombre, sino que son adquiridos; se han desarrollado y se desarrollan históricamente.

En el desarrollo de los instrumentos "materiales" científicos, que se inicia históricamente con el advenimiento del método experimental, se ha desarrollado una ciencia particular, la de los instrumentos, estrechamente vinculada al desarrollo general de la tecnología.

\subsection{Realidad}

Todos arrastramos de una manera implícita una forma de concebir la realidad del mundo que nos rodea, la mayoría de las veces es pensada como lo que está, frente a nosotros, ya dado como tal; a lo que podemos acercarnos, percibir, clasificar, ordenar, interpretar; pero estas acciones no la constituyen sino se acercan a lo dado como cosas que está ahí independiente de nuestra mirada.

Esta manera de acercarse al mundo natural y social está mostrando una concep- 


\section{Ridberth M. Ramírez Miranda}

ción enraizada en nuestra cultura, que no es "esencialmente humana", sino producto de una lógica, la que hemos heredado y a la que es posible cuestionar como modo único de concebir la realidad tanto del mundo natural como histórico-social.

Entonces, para pensar la constitución de la realidad en el sujeto y en el colectivo social, se vuelve necesario comenzar por el inicio de la psique humana y su transformación hasta advenir en individuo social; o sea hasta su acceso a ser alguien para quien existirán otros individuos, objetos, una sociedad, instituciones, un mundo; es decir, la emergencia del individuo social como coexistencia, realizada de una realidad privada y de una realidad pública o común.

Implica la creación del colectivo social que definirá un mundo, las cosas que en él se encuentran, las relaciones de esas cosas entre sí y con los individuos que habitan ese mundo; en cada momento confluyen situaciones (políticas, económicas, sociales) subjetivas que van plasmando una configuración a la que llamamos realidad y que es instituida como conjunto de significaciones imaginarias. La sociedad instituye en cada momento un mundo como su mundo o como el mundo.

Planteamos una estructura elemental para concebir lo social, donde el lector podrá ajustarla para analizar una realidad concreta, que legitime su investigación:

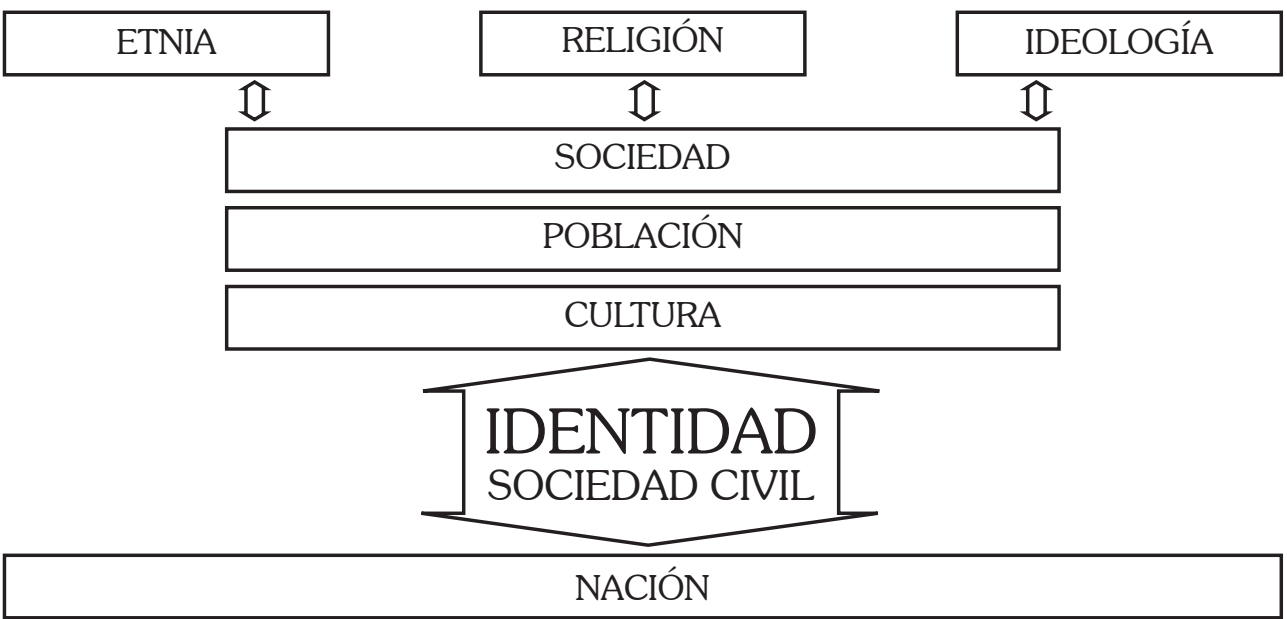




\section{Estructuraciones para analizar realidades complejas}

\section{EL CRITERIO DE LA CIENCIA Y LA TECNOLOGÍA}

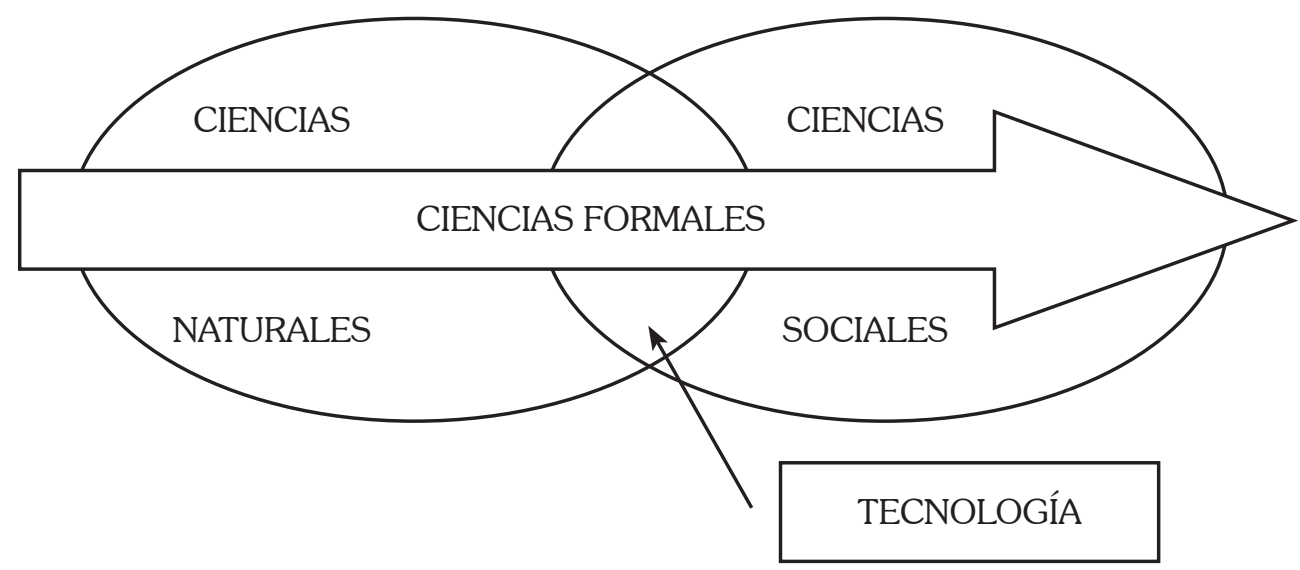

\subsection{Ciencias}

Es el conocimiento sistematizado, elaborado mediante observaciones, razonamientos y pruebas metódicamente organizadas. La ciencia utiliza diferentes métodos y técnicas para la adquisición y organización de conocimientos sobre la estructura de un conjunto de hechos objetivos y accesibles, basada en un criterio de verdad y una evolución permanente.

La aplicación de los métodos y conocimientos conduce a la generación de más conocimiento objetivo en forma de predicciones concretas, cuantitativas y comprobables referidas a hechos observables pasados, presentes y futuros. Con frecuencia, esas predicciones pueden formularse mediante razonamientos y estructurarse como reglas o leyes generales, que dan cuenta del comportamiento de un sistema y predicen cómo actuará dicho sistema en determinadas circunstancias.

Dentro de las ciencias, la ciencia experimental se ocupa solamente del estudio del universo natural ya que, por definición, todo lo que puede ser detectado o medido forma parte de él. Los científicos se ajustan a un cierto método, el método científico, un proceso para la adquisición de conocimiento empírico. A su vez, la ciencia puede diferenciarse en ciencia básica y aplicada, siendo esta última la aplicación del conocimiento científico a las necesidades humanas y al desarrollo tecnológico. 


\section{Ridberth M. Ramírez Miranda}

Consecuentemente, presentamos el esquema de clasificación planteado por el epistemólogo alemán Rudolf Carnap quien fue el primero en dividir a la ciencia en:

- Ciencias Formales. Estudian las formas válidas de inferencia: Lógica - Matemática. Por eso no tienen contenido concreto, es un contenido formal en contraposición al resto de las ciencias fácticas o empíricas.

- Ciencias Naturales. En ellas se encuadran las ciencias naturales que tienen por objeto el estudio de la naturaleza. Siguen el método científico: Astronomía - Biología - Física - Química - Geología - Geografía física.

- Ciencias Sociales. Son todas las disciplinas que se ocupan de los aspectos del ser humano - cultura y sociedad- El método depende de cada disciplina particular: Antropología - Ciencia política - Demografía- Economía - Historia - Psicología - Sociología - Geografía humana - Trabajo social

Cada ciencia, y aun cada investigación concreta generan su propio método de investigación, que está ligado a lo que los científicos utilizan el término modelo para referirse a una descripción de algo, especialmente una que pueda ser usada para realizar predicciones que puedan ser sometidas a prueba por experimentación u observación.

Es el caso de las Ciencias Sociales, donde los fenómenos no se pueden repetir controlada y artificialmente (que es en lo que consiste un experimento), sino que son, por su esencia, irrepetibles; de manera que el concepto de método científico aplicado a estas ciencias significaría un proceso de conocimiento caracterizado por el uso constante e irrestricto de la capacidad crítica de la razón, que busca establecer la explicación de un fenómeno ateniéndose a lo previamente conocido.

\subsection{Tecnología}

Tecnología es el conjunto de habilidades que permiten construir objetos y máquinas para adaptar el medio y satisfacer nuestras necesidades, es decir, conjunto de cono- 


\section{Estructuraciones para analizar realidades complejas}

cimientos formado por la triada: arte, técnica u oficio. La actividad tecnológica influye en el progreso social y económico.

Conjunto de teorías y de técnicas que permiten el aprovechamiento práctico del conocimiento científico. Esta acepción asimila la tecnología a ciencia aplicada o tecnociencia, lo que solo es válido para algunas tecnologías, las basadas en conocimientos científicos.

Algunas de las tecnologías actuales más importantes, como la electrónica, consisten en la aplicación práctica de las ciencias (en ese caso el electromagnetismo y la física del estado sólido). Sin embargo, no todas las tecnologías son ciencias aplicadas. Tecnologías como la agricultura y la ganadería precedieron a las Ciencias Biológicas en miles de años, y se desarrollaron de modo empírico, por ensayo y error (y por ello con lentitud y dificultad), sin necesidad de conocimientos científicos.

Las tecnologías han sido usadas para satisfacer necesidades esenciales (alimentación, vestimenta, vivienda, protección personal, relación social, comprensión del mundo natural y social), para obtener placeres corporales y estéticos (deportes, música, hedonismo en todas sus formas) y como medios para satisfacer deseos (simbolización de estatus, fabricación de armas y toda la gama de medios artificiales usados para persuadir y dominar a las personas).

No podemos dejar de mencionar a Joseph Alois Schumpeter, "quien es uno de los pocos economistas que asignó a las tecnologías un rol central en los fenómenos económicos. En sus obras, señala que los modelos clásicos de la economía no pueden explicar los ciclos periódicos de expansión y depresión, como los de Kondratiev, que son la regla más que la excepción. El origen de estos ciclos, según Schumpeter, es la aparición de innovaciones tecnológicas significativas (como la introducción de la iluminación eléctrica domiciliaria por Edison o la del automóvil económico por Ford) que generan una fase de expansión económica. La posterior saturación del mercado y la aparición de empresarios competidores cuando desaparece el monopolio temporario que da la innovación, conducen a la siguiente fase de depresión. El término empresario schumpeteriano es hoy corrientemente usado para designar a los empresarios innovadores que hacen crecer su industria gracias a su creatividad, capacidad organizativa y mejoras de eficiencia". 


\section{Ridberth M. Ramírez Miranda}

\section{CRITERIO DE LA VINCULACIÓN Y ALIANZAS PARA EL DESARROLLO}

El criterio: UNIVERSIDAD, EMPRESA Y ESTADO, implica la necesidad del conocimiento científico-tecnológico y la constitución de redes-alianzas sociales-empresariales con el Estado.

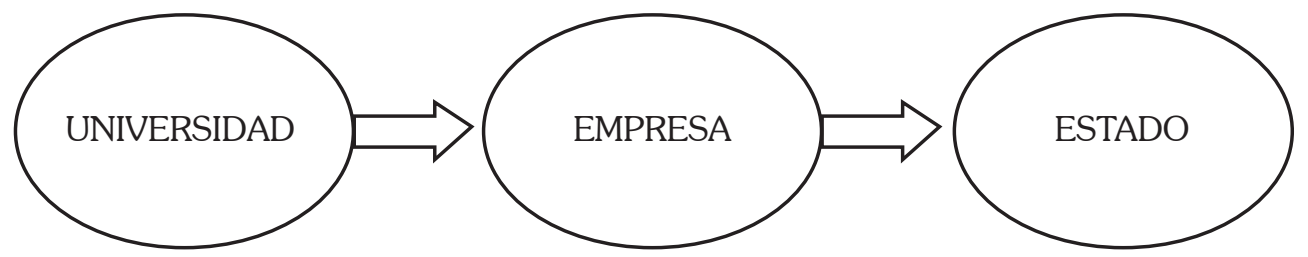

Nociones recientes reivindican el papel promotor para el Estado, fundamentalmente en todos sus niveles macro, meso y micro, dinamizando y garantizando oportunidades de surgimiento, expansión y consolidación de la producción nacional.

La globalización permite el fortalecimiento del capital transnacional, abriéndole amplias posibilidades para movilizarse y multiplicarse en todos los confines de la tierra. En nuestra realidad la visión estratégica ante la disminución de maniobrabilidad política y económica ha afectado al Estado haciendo que le sea difícil garantizar un nivel de calidad de vida aceptable para todos sus habitantes y además, permitir un desarrollo armónico para el país.

Es aquí donde se centra el debate, entre los que creen que el mercado por sí solo regula las relaciones sociales y los que están convencidos de un papel más activo del Estado. El hecho de brindar infraestructura o servicios básicos, es importante para garantizar las inversiones, pero hoy en día, las dinámicas existentes demandan un papel más activo, abandonando el tradicional enfoque keynesiano, pero asumiendo una función determinante en la superación de las fallas del mercado, la inducción de pactos sociales y la promoción del desarrollo ambientalmente sostenible.

El fenómeno de la globalización toca a las universidades, persuadiéndolas de hacer un mayor esfuerzo en entender y desarrollar conocimiento útil y pertinente para los procesos productivos. Siendo así, es indefectible una alianza social entre las 


\section{Estructuraciones para analizar realidades complejas}

\section{universidades, las empresas y el Estado para incorporar conocimiento, inno- vación y diseñar e implementar estrategias coherentes para competir en los mercados nacionales y mundiales.}

Para ello, es necesario promover vinculaciones y alianzas entre la Universidad Empresa - Estado, donde un grupo de académicos, empresarios y funcionarios se reúnan para analizar y proponer la alternativa más viable para la producción, difusión y apropiación del conocimiento científico para fines productivos y sociales.

La iniciativa debe ser fundamentalmente conformar equipos científicos con representantes de las universidades, las empresas y el Estado, para proponer estrategias que contribuyan al desarrollo de la ciencia y la tecnología en el país.

En todos los modelos de desarrollo científico del mundo, el Estado es el principal comprador de conocimiento; por tanto el gobierno, debe tener el compromiso de apoyar a las investigaciones en las universidades, y asimismo, las universidades deben ser reconocidas como productoras de conocimiento y no como vendedoras de consultorías solamente.

Los proyectos de investigación de las universidades se enmarcan solo en lo académico, peor aun, cuando las supuestas investigaciones solo son análisis efectuados con instrumentos hartamente conocidos, que no responden ni siquiera a las necesidades del sector productivo, que las empresas no aprovechan la capacidad de conocimiento de los centros de investigación para el mejoramiento de sus procesos y que el Estado no incentivaba la unión de estas instituciones para trabajar de manera conjunta en proyectos de gran impacto.

La universidad no ha diseñado una estrategia para romper ese aislamiento; por eso, se debe crear vinculaciones y alianzas entre los tres entes: Universidad-EmpresaEstado, cuya estrategia debe promover las relaciones entre estos actores con el fin de generar proyectos asociativos basados en la investigación aplicada, la innovación y la gestión del conocimiento.

De esta manera, el vínculo Universidad-Empresa-Estado alcanzaría una dimensión global y se constituiría en un instrumento importante que contribuiría a mejorar la pro- 


\section{Ridberth M. Ramírez Miranda}

ductividad de las empresas, incentivaría a los investigadores para que generen proyectos de impacto social, económico y productivo; pero, sobre todo, permitirá la explotación de las potencialidades del país para que puedan avanzar en el camino hacia su desarrollo sostenido.

En este sentido presentados las estructuraciones para cada ente.

\subsection{Universidad}

Aunque parezca obvio, también debe destacarse que la investigación en las universidades debe ser universal. El término universidad se justifica precisamente porque se refiere a un estado de la mente por arriba del aprendizaje de recetas o de meros hechos. Lo que es importante en la investigación es que el investigador comprenda lo que está investigando o lo que está haciendo, que lo conceptualice, que pueda dominarlo bajo distintos ángulos, que pueda evaluarlo y que tome tina posición crítica en relación a lo que investiga.

Al crecer las universidades, también se han diversificado las carreras que en ellas se enseñan, se han transformado en grandes instituciones en las que se enseñan tanto las disciplinas tradicionales como otras que surgen de la integración de disciplinas ya existentes, como en el caso de la bioquímica, o de la profesionalización de oficios, como el diseño gráfico, o del avance de conocimientos mediante la investigación, como la biotecnología. Los logros en el campo de la investigación científica y aplicada se han diversificado como las carreras.

El concepto de verdad es fundamental para la investigación en las universidades. Es cierto que no todos los investigadores tienen la misma idea de lo que es la verdad; pero, generalmente, la saben distinguir y es uno de los valores que más aprecian en el campo de la investigación y también en las relaciones cotidianas con otros miembros de la comunidad científica. Los logros del conocimiento mediante la investigación lo consideramos objetivos cuando los entendemos de la misma manera, cuando los podemos transmitir y cuando los podemos reproducir de manera independiente. 


\section{Estructuraciones para analizar realidades complejas}

El incorporar una visión crítica, en el sentido positivo de la crítica, a los programas de investigación y a las actividades de investigación que se desarrollan en las universidades resulta realmente difícil, en especial en las Ciencias Naturales, ya que se ve más difícil criticar a la naturaleza que a los procesos sociales. Pero aun en las Ciencias Naturales es posible tomar una posición crítica sobre sus métodos y su papel en la sociedad.

Consecuentemente, la universidad desempeña un papel muy importante en la sociedad como conciencia crítica de la misma. Su autonomía y la libertad académica le permiten desempeñar este papel, más ahora que las democracias se han consolidado en la mayoría de los países del mundo. Sin embargo, sobre el ejercicio de la función crítica en las universidades latinoamericanas, siempre han predominado las expresiones políticas e ideológicas sobre otras que pudieran ser a la larga, más orgánicas y efectivas. Desde luego que la crítica de la universidad será tomada en cuenta en la medida en que se fundamente en un trabajo de investigación serio y comprometido.

Es necesario asumir una lógica estructural para abordar su importancia.

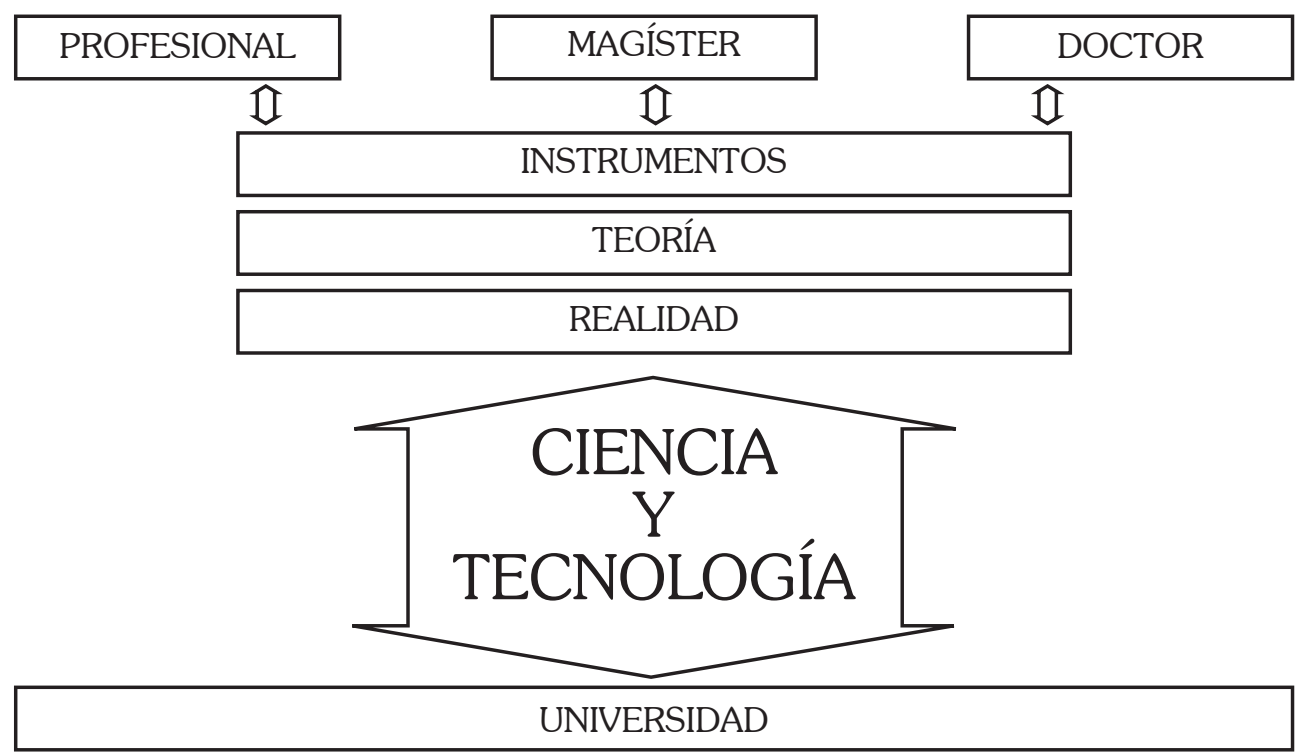




\section{Ridberth M. Ramírez Miranda}

\subsection{Empresa}

La empresa, además de ser una célula económica, es una célula social. Está formada por personas y para personas. Está insertada en la sociedad a la que sirve y no puede permanecer ajena a ella. La sociedad le proporciona la fuerza de trabajo y el mercado de consumidores; la educación de sus obreros, técnicos y profesionales; los medios de comunicación y la llamada infraestructura económica; la empresa recibe mucho de la sociedad y existe entre ambas una interdependencia inevitable.

La liberalización del mercado y el fin de la intervención por parte del Estado supusieron una mayor intervención de la empresa en el gobierno a nivel nacional y global. La inversión extranjera directa constituye el método preferido de transferencia de capital y de transmisión de capacidades y tecnología y se considera a la empresa como motor de crecimiento en todos los ámbitos, donde la universidad aparentemente no aparece pero implícitamente si como proveedora de ciencia y tecnología. Sin embargo, como consecuencia de todo ello, aumentan las expectativas de la sociedad civil y se exige una mayor intervención de la empresa en ámbitos tan variados como la seguridad del empleo, los subsidios, la normativa relativa al medio ambiente y la transparencia en las transacciones empresariales.

La estrategia y la política empresarial se encuentran con problemas adicionales en el momento de reconciliar las presiones que emanan de la competitividad global y la transformación social. Se requieren nuevas estrategias, la promoción de instituciones de mediación que creen vínculos entre la universidad, la empresa y la sociedad, y la obtención de un entorno político más favorable para optimizar y distribuir los beneficios que emanan de la inversión y el crecimiento; la cohesión social, la cantidad y calidad de puestos de trabajo existentes en las redes de producción global, y la creciente adopción de códigos de conducta y directrices empresariales por cuestiones de ética.

La transformación de la industria resulta más efectiva, y la cohesión social menos afectada, cuando reciban el apoyo de las universidades como ente científico-tecnológico y de las redes políticas que conectan una gran selección de organizaciones intermediarias privadas, semiprivadas y públicas. Los que se magnificarían mediante el estímulo y la coordinación de la cooperación entre las universidades, las empresas y las agencias 


\section{Estructuraciones para analizar realidades complejas}

públicas, contribuyendo a desarrollar una nueva competencia industrial y a aumentar la oportunidades de empleo.

Si los códigos de conducta empresariales constituyen la solución evidente a los problemas sociales de los consumidores y la opinión pública, otra respuesta igual de extendida es la participación de las universidades en el proceso productivo y la proliferación de iniciativas sociales por parte de las empresas, las cuales abarcan una amplia gama de programas de participación voluntaria de la comunidad, como: iniciativas de formación y educación de los trabajadores locales, simplificación del acceso al mercado de trabajo por parte de los grupos desfavorecidos, promoción de la cohesión social, apoyo a las organizaciones de carácter comunitario, etc.

Las grandes empresas saben lo importante que es digerir bien los cambios y la tecnología. No obstante, desde la burbuja de los puntos.com que generó el inicio de la eclosión de internet, los cambios asociados a la tecnología y el conocimiento son costosamente digeribles. La sociedad del conocimiento en la empresa, ha generado nuevas reglas, nuevas mentalidades

La globalización del conocimiento a través de internet está originando cambios de una entidad y rapidez desconocida. Algunas empresas líderes de la última generación han asumido la premisa básica de la nueva gestión de la economía del conocimiento: El conocimiento que se comparte crece". La red hace posible el que concurso de decenas de miles de mentes puedan dedicarse, sin apenas coordinación a analizar, pensar, desarrollar, avanzar en una cuestión dada.

Internet, la sociedad de la información y la economía del conocimiento han supuesto, suponen y supondrán cambios muy relevantes para las empresas; pero internet no va a ser el único salto tecnológico. No hay que tener miedo al intercambio de ideas. Olvidar el viejo precepto de "la información es poder" y sustituirlo por el de "el conocimiento que se comparte cree", es vital para el futuro de la empresa.

Lamentablemente, en muchas de las empresas tradicionales, la cúpula directiva se recrea en discusiones interminables y en estrategias personales destinadas más a dar "autobrillo" personal que soluciones operativas. Muchas empresas están destinadas a 


\section{Ridberth M. Ramírez Miranda}

fracasar en el nuevo dogma de la sociedad del conocimiento: de trabajar compartiendo conocimientos y en el desarrollo de nuevas actitudes y aptitudes frente a los grandes retos del futuro en la empresa.

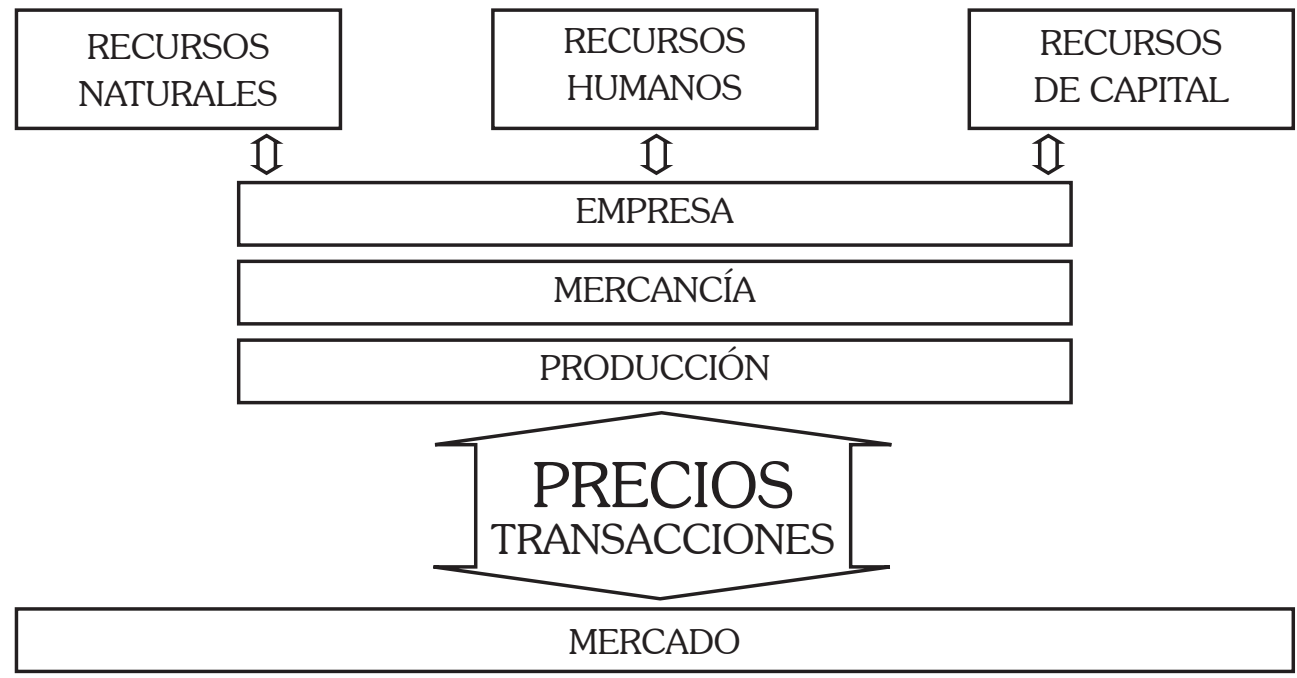

A propósito de la tecnología de la empresa, un país con grandes recursos naturales será pobre si no tiene las tecnologías necesarias para su ventajosa explotación, lo que requiere una enorme gama de tecnologías de infraestructura y servicios esenciales. Asimismo, un país con grandes recursos naturales bien explotados tendrá una población pobre si la distribución de ingresos no permite a esta un acceso adecuado a las tecnologías imprescindibles para la satisfacción de sus necesidades básicas. En la actual economía capitalista, el único bien de cambio que tiene la mayoría de las personas para la adquisición de los productos y servicios necesarios para su supervivencia es su trabajo. La disponibilidad de trabajo, condicionada por las tecnologías, es hoy una necesidad humana esencial.

Si bien las técnicas y tecnologías también son parte esencial del trabajo artesanal, el trabajo fabril introdujo variantes en la organización y realización del trabajo de producción. El alto costo de las máquinas usadas en los procesos de fabricación masiva, tuvo como consecuencia que el trabajador perdiera el control de su modo de trabajar. 


\section{Estructuraciones para analizar realidades complejas}

\subsection{Estado}

El Estado es un concepto político que se refiere a una forma de organización social soberana y coercitiva, formada por un conjunto de instituciones que tiene el poder de regular la vida sobre un territorio determinado; es el conjunto de instituciones que poseen la autoridad y potestad para establecer las normas que regulan la sociedad, teniendo soberanía interna y externa sobre un territorio determinado.

El Estado es una organización que reclama para sí -con éxito- el "monopolio de la violencia legítima"; por ello, dentro del Estado, se incluyen instituciones tales como las fuerzas armadas, la administración pública, los tribunales y la policía, asumiendo pues el Estado las funciones de defensa, gobernación, justicia, seguridad y otras como las relaciones exteriores. Asimismo, como evolución del concepto, se ha desarrollado el "Estado de Derecho", por el que se incluyen dentro de la organización estatal aquellas resultantes del imperio de la ley y la división de poderes (ejecutivo, legislativo y judicial) y otras instituciones autónomas, pero propias del Estado.

No debe confundirse con el concepto de Gobierno, que sería solo la parte generalmente encargada de llevar a cabo las funciones del Estado delegando en otras instituciones sus capacidades. El Gobierno también puede ser considerado como el conjunto de gobernantes que, temporalmente, ejercen cargos durante un período de tiempo limitado dentro del conjunto del Estado, pero que en el fondo son los responsables de lo que pasa en el Estado

Tampoco equivale totalmente al concepto de carácter más ideológico, de "Nación", puesto que se considera posible la existencia de naciones sin Estado y la posibilidad de que diferentes naciones o nacionalidades se agrupen en torno a un solo Estado. Comúnmente los Estados forman entes denominados "Estado-Nación" que aúnan ambos conceptos, siendo habitual que cada nación posea o reivindique su propio Estado.

El Estado es uno de los pocos seres institucionales que sobreviven sin una evolución importante en su estructura y funcionamiento, con excepción de su crecimiento. El enfoque crítico difiere además entre el institucionalismo y el clasismo como factor determinante de la naturaleza del Estado. Algunas, concepciones como anarquismo consideran conveniente la total desaparición de los Estados, en favor del ejercicio so- 


\section{Ridberth M. Ramírez Miranda}

berano de la libertad individual a través de asociaciones y organizaciones libres. Otras concepciones aceptan la existencia del Estado, con mayor o menor autoridad o potestad, pero difieren en cuanto cual debiera ser su forma de organización y el alcance de sus facultades.

Evidentemente, es un tema que se discute permanente, pero la presente estructura podría servirnos en los procesos de investigación relacionados con el tema: Universidad, Empresa, Estado.

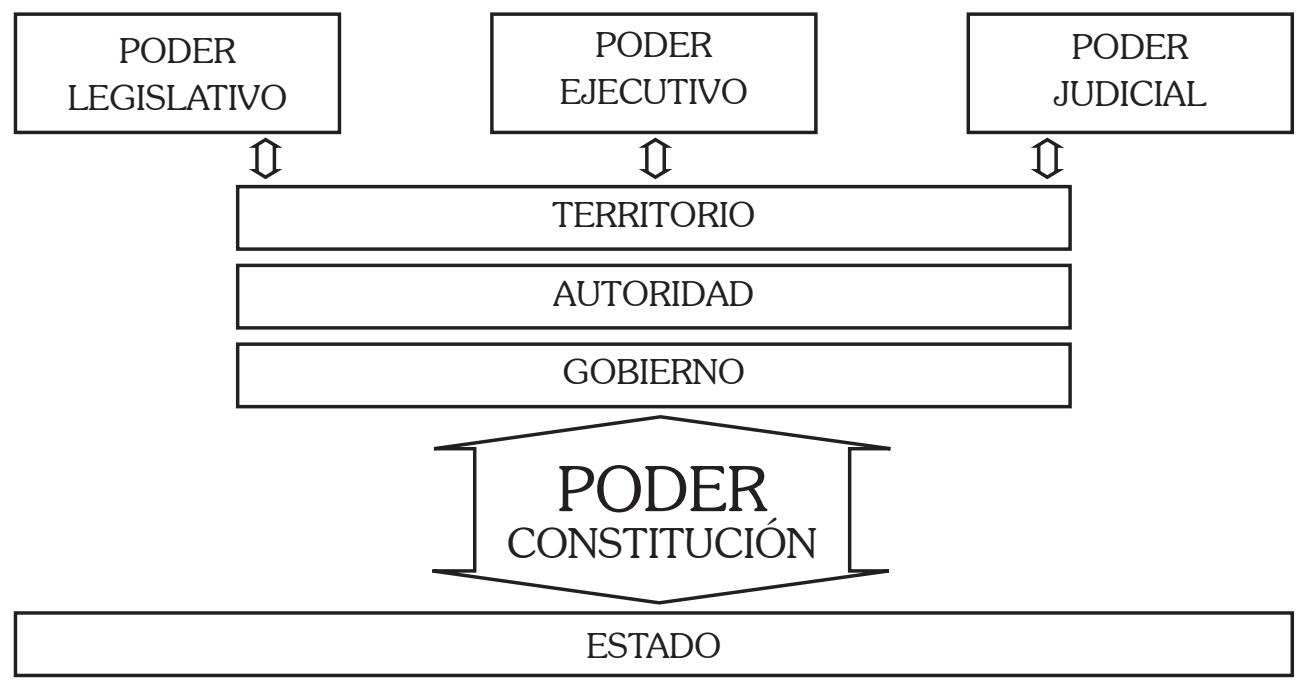

Finalmente, presento solo la estructura que podría ser un tema de investigación, que podría desarrollar la universidad.
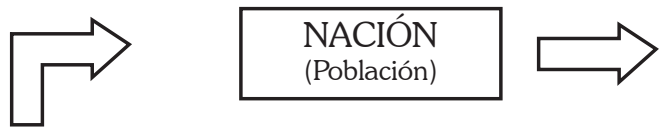

PROYECTO SOCIAL

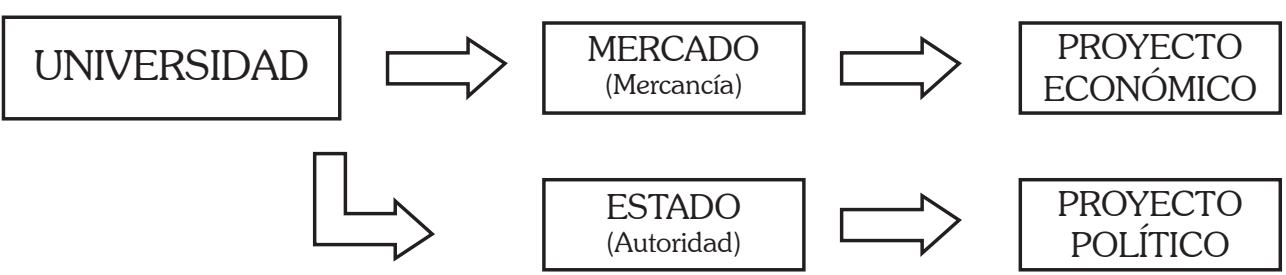




\section{Estructuraciones para analizar realidades complejas}

\section{BIBLIOGRAFÍA}

Bonvecchio, Claudio. El Mito de la Universidad. México, Siglo XXI Editores-UNAM, 1991.

Brunner, José Joaquín. Educación Superior en América Latina: Cambios y Desafíos. Fondo de Cultura Económica, Santiago de Chile, 1990.

C. Castoriadis. "La institución imaginaria de la sociedad" Vol. I y II Ed. Tusquets.

Castrejón Diez, Jaime. El Concepto de Universidad. Ediciones Océano, México, 1982.

Fernández Alvarez, Manuel. Historia en La Universidad de Salamanca. Ediciones Universidad de Salamanca, Salamanca, 1991.

Gil Antón, Manuel et al. Académicos: Un Botón de Muestra. México, Universidad Autónoma Metropolitana, 1992.

González Cuevas, Oscar. "Algunos Comentarios sobre Investigación y Docencia". En: Educación Química. Vol. 3, N.ำ 1992.

Ilya Prigogine. “¿El fin de la ciencia?”. En: Nuevos Paradigmas, Cultura y Subjetividad. Edit. Paidos.

Latapí, Pablo. "Algunas Tendencias de las Universidades Latinoamericanas. Problemas Seleccionados y Perspectivas". En: "Seminario sobre Nuevas Tendencias y Responsabilidades para las Universidades en Latinoamérica”. México, UDUAL, 1978.

Ortega y Gasset, José. "Misión de la Universidad". En: Revista de Occidente. Madrid, Alianza Editorial, 1930. 